Published in final edited form as:

Eur J Cancer Prev. 2014 November ; 23(6): 502-505. doi:10.1097/CEJ.0000000000000029.

\title{
Maternal Exercise During Pregnancy Reduces Risk of Mammary Tumorigenesis In Rat Offspring
}

\author{
Ignacio Camarillo ${ }^{1}$, Leon Clah ${ }^{1}$, Wei Zheng ${ }^{2}$, Xuanzhu Zhou ${ }^{2}$, Brienna Larrick ${ }^{2}$, Nicole \\ Blaize $^{4}$, Emily Breslin ${ }^{4}$, Neal Patel ${ }^{1}$, Diamond Johnson ${ }^{1}$, Dorothy Teegarden ${ }^{2}$, Shawn S. \\ Donkin $^{3}$, Timothy P. Gavin ${ }^{4}$, and Sean Newcomer ${ }^{4}$ \\ ${ }^{1}$ Department of Biological Sciences, 915 W. State Street, Purdue University, West Lafayette, IN \\ 47907
}

${ }^{2}$ Department of Nutrition Science, 700 W. State Street, Purdue University, West Lafayette, IN 47907

${ }^{3}$ Department of Animal Sciences, 915 W. State Street, Purdue University, West Lafayette, IN 47907

${ }^{4}$ Department of Health and Kinesiology, 800 W. Stadium Avenue, Purdue University, West Lafayette, IN 47907

\begin{abstract}
Breast cancer is the most common cancer among women. Emerging research indicates that modifying lifestyle factors during pregnancy may convey long-term health benefits to offspring. This study was designed to determine whether maternal exercise during pregnancy leads to reduced mammary tumorigenesis in female offspring. Pregnant rats were randomly assigned to exercised and sedentary groups, with the exercised group having free access to a running wheel and the sedentary group housed with a locked wheel during pregnancy. Female pups from exercised or sedentary dams were weaned at 21 days of age and fed a high fat diet without access to a running wheel. At 6 weeks, all pups were injected with the carcinogen N-methyl-Nnitrosourea (MNU). Mammary tumor development in all pups was monitored for 15 weeks. Pups from exercised dams had a substantially lower tumor incidence (42.9\%) compared to pups from sedentary dams $(100 \%)$. Neither tumor latency nor histological grade differed between the two groups. These data are the first to demonstrate that exercise during pregnancy potentiates reduced tumorigenesis in offspring. This study provides an important foundation towards developing more effective modes of behavior modification for cancer prevention.
\end{abstract}

\section{Keywords}

fetal programming; free wheel running; prevention; breast cancer and add exercise

Corresponding Author: Dorothy Teegarden, Purdue University, 700 W. State Street, West Lafayette, IN 47907; teegarden@ purdue.edu; PH: 765-494-8246; FAX: 765-494-0906.

Conflict of Interest Statement: No potential conflicts of interest were disclosed by the authors. 


\section{Introduction}

Physical activity plays a significant role in the prevention of chronic diseases. Increased participation in physical activity decreases the risk for the development of cardiovascular disease, diabetes, and cancers [1]. Emerging evidence demonstrates that maternal exercise during pregnancy may play a role in the prevention of these chronic diseases in the offspring through fetal programming in utero [2]. However, the relationship between maternal exercise during pregnancy and susceptibility of offspring to develop breast cancer has not been explored.

In developmental the mammary gland is more susceptible to carcinogenesis during puberty, pregnancy and post-menopause, periods when significant mammary tissue remodeling is evident and environmental insults and/or genetic aberrations may lead to epithelial transformation. Such windows also coincide with optimal periods for behavior modification towards cancer prevention. Exercise during adolescence or menopause is associated with decreased breast cancer risk in an individual [3]. Additional opportunities to alter risk may exist during the prenatal period of mammary development. Indeed evidence linking dietary and estrogenic exposure in pregnancy to increased risk of mammary cancer in multiple generations of offspring $[4,5]$. The current study examined the impact of maternal exercise during pregnancy on mammary tumorigenesis in the offspring.

\section{Materials \& Methods}

\section{Animals}

Virgin female Sprague Dawley rats were utilized. Upon arrival, all dams were housed individually with access to a running wheel in order to become acclimated to their environment and running wheel. On day 4, the exercise wheels were locked and male rats were housed with the dams for breeding purposes. All animal experiments were approved by the Purdue Animal Care and Use Committee.

\section{Intervention}

Following breeding, dams were randomly assigned into sedentary $(n=4)$ and exercised $(n=$ 7) experimental groups. All dams were housed individually in running wheel cages. The exercise group had access to an unlocked running wheel and the sedentary group housed with a locked running wheel during pregnancy. All dams were fed a standard chow (Harlan Rodent Diet 2018, Indianapolis, IN, USA) ad libitum and weighed daily. Total distance (km) was recorded daily for all exercise dams using a magnetic sensory system (Rat Activity Wheel, Lafayette Instrument, Lafayette, IN, USA or Schwinn 17 Function Bike Computer, Pacific Cycle, Madison, WI, USA). Once the dams gave birth to the offspring, the exercise wheels were locked and the pups continued to be housed with their birth mother until they were weaned at 21 days of age.

\section{Experimental Groups}

Measurements of litter size and weight were taken 1 day after birth. Following weaning, 1 female offspring from all exercise dams, 1 female offspring from a sedentary dam and 2 
female offspring from each of the 3 remaining sedentary dams were randomly selected and assigned to offspring from exercise $(n=7)$ and offspring from sedentary $(n=7)$ experimental groups. Throughout the duration of the experiment offspring were individually housed without access to a running wheel and fed a high fat diet comprised of $20 \%$ protein, 45\% fat, and 35\% carbohydrate (D12451, Open Source Diets, New Brunswick, NJ, USA).

\section{Tumor Induction and Assessment}

At 1.5 months following weaning, the pups from exercised and sedentary dams were given a single injection of N-Methyl-N-nitrosourea (MNU) intraperitoneally at $50 \mathrm{mg} / \mathrm{kg}$ to induce tumors in the mammary glands, and maintained on their respective diets. The pups were inspected bi-weekly for developing tumors which were palpated by hand and measured with digital calipers on three axes. The sizes of the tumors were documented as $\mathrm{mm}^{3}$. Animals were sacrificed at 4 months post MNU injection. A sample from each tumor of all animals was collected and subjected to histological verification for tumor grade. For histological evaluation, mammary tumor subsamples were fixed in $10 \%$ neutral formalin for paraffin embedding. Tumor grade was histologically classified by the Purdue Veterinary Medicine Histopathology laboratory. Tumor onset data was analyzed using the Kaplan-Meier method and Proc Lifetest in SAS.

\section{Statistical Analysis}

Descriptive and univariate statistics were assessed for all variables. Student's t-test was used to compare groups. Litter characteristics were analyzed using a repeated measures analysis of variance (ANOVA) where dam was nested in intervention. Tumor onset data were analyzed as right-censored survival data using the Kaplan-Meier method and Proc Lifetest in SAS. Each group was compared using the log-rank test. Results are expressed as mean $\pm \mathrm{SE}$. Values were considered significant at $\mathrm{P} \unlhd \mathbf{} \mathbf{0 5}$.

\section{Results}

The distance dams ran during pregnancy is shown in Figure 1A. Exercised dams ran on average $2.0 \pm 0.2 \mathrm{~km} / \mathrm{d}$ during pregnancy. There was no significant difference in litter weight between the pups from sedentary dams and the pups from exercised dams $(103.2 \pm 3.7 \mathrm{~g}$ vs. $93.1 \pm 4.1 \mathrm{~g}$, respectively). The litter size of sedentary dams was significantly greater (15.5 \pm 0.9 pups/dam) compared to that of the exercised dams (13.7 \pm 0.3 pups/dam, $\mathrm{P}=0.04$ ). The weight of the sedentary dams was not different compared to the exercised dams at any time (Figure 1B).

The incidence of mammary tumors was lower in pups from exercised (42.9\%) compared to those from sedentary (100.0\%) dams (Figure 2D). In pups that did develop tumors, the average tumor latency period was not significantly different in pups from exercised (61.7 \pm 1.2 days) compared to pups from sedentary (66.5 \pm 9.1 days) dams (Figure 2D). Histological analysis of mammary tumors revealed that tumor grade was not different in the pups from sedentary versus exercised dams (Figure 2E). Specifically, pups with tumors from both sedentary and exercised dams developed mammary adenocarcinomas. There was no difference in the body weights (Figure 2A), average tumor frequency (Figure 2B), or 
average volume per animal that developed tumors (Figure 2C) between the pups from the exercised dams compared to the pups from the sedentary dams.

\section{Discussion}

In summary, these data are the first to demonstrate that maternal exercise during pregnancy imparts substantial protection against mammary tumorigenesis in female offspring. As anticipated [6], there was a $100 \%$ incidence of mammary tumors in pups from sedentary mothers, and an unprecedented reduction to $42 \%$ incidence in pups from exercised dams. This data suggests that maternal exercise during pregnancy modulates early life events to potentiate protection from breast cancer in offspring even following powerful carcinogenic exposure.

A growing body of epidemiological data clearly demonstrates that daily participation in moderate to vigorous physical activity can reduce the incidence of breast cancer by 25-30\% [7], but the mechanism is unclear. From the current results, we can speculate that changes in maternal metabolism or factors released into circulation in response to exercise could impart robust anti-carcinogenic programming effects to the developing fetus. Healthy lifestyle choices during pregnancy, including proper nutrition and exercise, appear to promote offspring health that also persists beyond the neonatal period and may be mediated through changes in maternal metabolism, placental growth and vascularity, oxidative stress and endothelial cell function $[8,9]$. Future investigations will be needed to elucidate the mechanisms underlying the protective effects of maternal exercise during pregnancy on breast cancer susceptibility in offspring.

To our knowledge, this is the first report demonstrating that maternal exercise during pregnancy results in a significant reduction of mammary tumor incidence in female offspring. At this time, the underlying mechanisms that mediate the protective effect of maternal exercise on tumorigenesis are unclear, although various possibilities exist. These results provide an important first step towards identifying pregnancy as a potential time when maternal exercise effectively transmits substantial protection to offspring from cancer.

\section{Acknowledgments}

This project was supported by the National Institutes of Health, National Cancer Institute R25CA128770 and by a Project Development Team with the ICTSI NIH/NCRR Grant Number TR000006.

\section{References}

1. Booth FW. Reduced physical activity and risk of chronic disease: the biology behind the consequences. Eur J Appl Physiol. 2008; 102:381-390. [PubMed: 17987311]

2. Newcomer SC, Taheripour P, Bahls M, Sheldon RS, Foust K, Cabot R. Impact of porcine maternal aerobic exercise training during pregnancy on endothelial cell function of offspring at birth. Journal of Developmental Origins of Health and Disease. 2012; 3(1):4-9. [PubMed: 25101806]

3. Friedenreich CM, Courneya KS, Bryant HE. Influence of physical activity in different age and life periods on the risk of breast cancer. Epidemiology. 2001; 12:604-612. [PubMed: 11679785]

4. de Assis S, Warri A, Cruz MI, Laja O, Tian Y, Zhang B, Wang Y, Huang TH, Hilakivi-Clarke L. High-fat or ethinyl-oestradiol intake during pregnancy increases mammary cancer risk in several generations of offspring. Nat Commun. 2012; 3:1053. [PubMed: 22968699] 
5. Cho K, Mabasa L, Bae S, Walters MW, Park CS. Maternal high-methyl diet suppresses mammary carcinogenesis in female rat offspring. Carcinogenesis. 2012; 33:1106-12. [PubMed: 22431720]

6. Thordarson G, Lee AV, Talamanted F. Growth and characterization of N-methyl-N-nitrosoureainduced mammary tumors in intact and ovariectomized rats. Carcinogenesis. 2001; 22(12):2039-47. [PubMed: 11751437]

7. Na HK, Oliynyk S. Effects of physical activity on cancer prevention. Ann N Y Acad Sci. 2011; 1229:176-83. [PubMed: 21793853]

8. Weissgerber TL, Wolfe LA, Davies GA. The role of regular physical activity in preeclampsia prevention. Med Sci Sports Exerc. 2004; 36:2024-31. [PubMed: 15570135]

9. Sankaralingam S, Jiang Y, Davidge ST, Yeo S. Effect of exercise on vascular superoxide dismutase expression in high-risk pregnancy. Am J Perinatol. 2011; 28:803-10. [PubMed: 21815126] 


\section{Brief Description}

The aim of the study was to determine the impact of maternal exercise on the risk of mammary cancer in the female offspring. Our novel results are the first to show that maternal exercise provides substantial protection from mammary cancer development in female offspring. These results are significant as they hold tremendous potential for identifying a simple and safe lifestyle intervention that could significantly reduce the risk for mammary cancer in subsequent generations. 


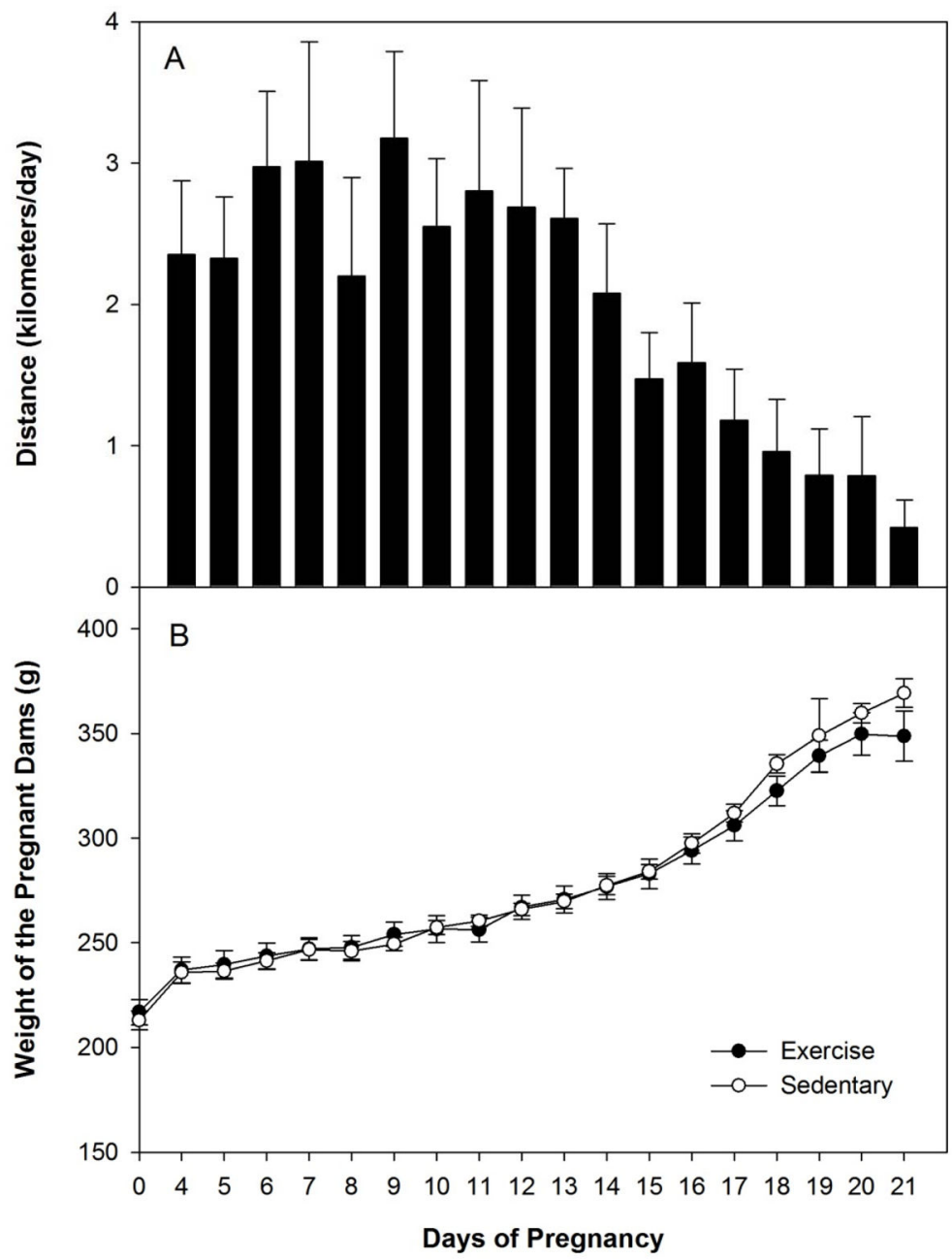

Figure 1. Maternal Exercise and Body Weight of Dams

Exercise dams were given free access to a running wheel throughout pregnancy. The average daily voluntary run distance in $\mathrm{km}$ are shown in A. The body weights of pregnant dams in both exercise and sedentary groups by day of pregnancy are shown in B. 


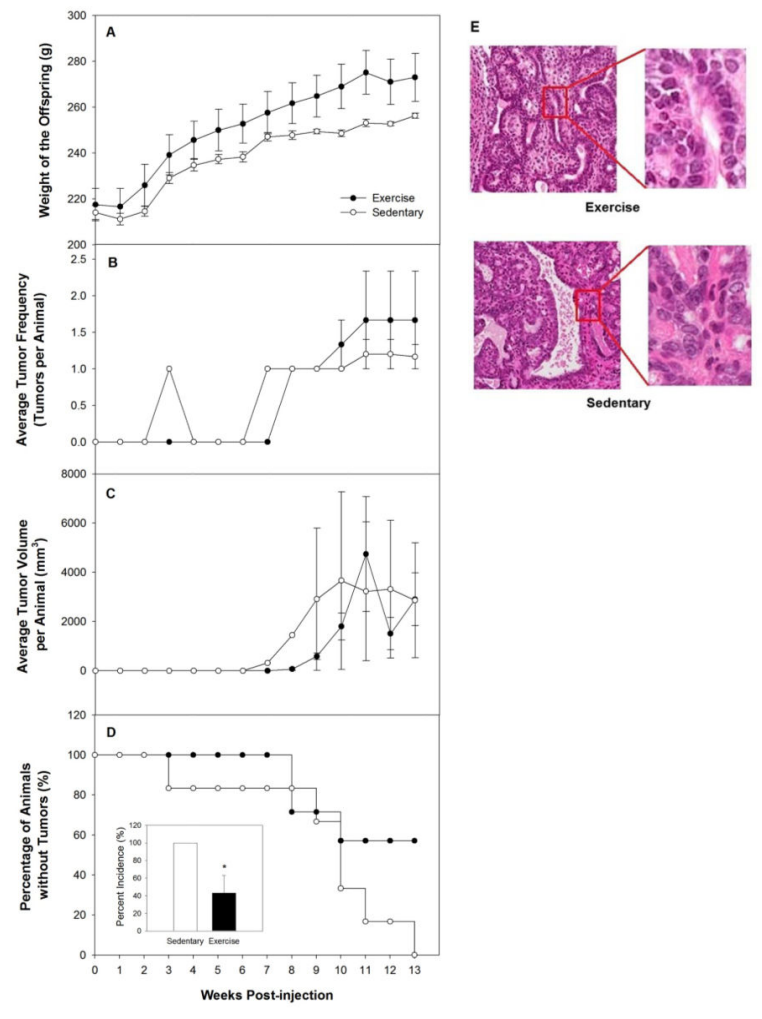

Figure 2. Mammary Tumor Development in Offspring

Pups from exercise (Exercise) and sedentary (Sedentary) dams were injected with MNU at 1.5 months following weaning. Body weights of the offspring (A), average tumor frequency (B) and average tumor volume (C) per animal that developed tumors are shown by weeks post-injection. A modified Kaplan-Meier curve (D) shows mammary tumor onset and incidence in pups from exercised and sedentary dams, and the inset shows the final cumulative tumor incidence with the asterisk indicating significant difference $(\mathrm{p}<0.05)$. A representative image of the histological analysis of the tumors in the pups from the exercised dams compared to the pups from the sedentary dams is shown (E). 